\title{
COSMOLOGY AND FLUCTUATIONS IN THE RADIATION BACKGROUNDS
}

\author{
P. J. E. Peebles \\ Joseph Henry Laboratories \\ Princeton University \\ Princeton, NJ USA
}

\begin{abstract}
Four topics are discussed. First, measurements of the autocorrelation function of the optical extragalactic sky background check the possibility that there is an appreciable contribution from young galaxies or from stars well outside normal galaxies. Second, large-scale fluctuations in the mass distribution are probed by the anisotropies of the X-ray and $2.7 \mathrm{~K}$ backgrounds. Third, scattering by plasma in young galaxies could affect the primeval anisotropy of the $2.7 \mathrm{~K}$ background radiation. Fourth, production of the heavy elements is expected to yield background radiation with a characteristic and perhaps detectable angular distribution.
\end{abstract}

\section{INTRODUCTION}

Angular structures in the radiation backgrounds play a central role in the observational basis for cosmology, from the detection of individual objects to the measurement of the statistics of their distributions. I have selected for discussion examples that are timely because they are relevant to current debates in cosmology and because they seem to be good prospects for further research. I have attempted only to present the motivation from cosmology and explanations of the orders of magnitude, leaving details for the research papers.

I assume the standard general relativity Friedmann-Lemaitre cosmology. For simplicity I take the cosmological constant $\Lambda$ to be negligibly small, but where possible the scaling with the density parameter, $\Omega$, is indicated. ( $\Omega$ is the ratio of the present mean mass density to the density in the Einstein-de Sitter model with $\Lambda=0$, zero space curvature, and the observed Hubble constant.) In this set of models, the angle $\theta$ subtended by an object with proper physical diameter $d$ at high redshift $z$ is

$$
\theta=\frac{\Omega H d}{2 c}(1+z) .
$$

Hubble's constant will be written as

$$
H=100 h \mathrm{~km} \mathrm{~s}^{-1} \mathrm{Mpc}^{-1}, h \sim 0.67 \pm 0.08 .
$$

The estimate of the dimensionless parameter $h$ is from van den Bergh (1989).

I use only the simplest measure of background fluctuations, the autocorrelation function (or second central moment). If the sources of the background are clustered like galaxies, the second moment is determined by the galaxy two-point spatial correlation function, $\xi(r)$, which is defined by the joint probability of finding galaxies in both of the volume elements $d V_{1}$ and $d V_{2}$ at separation $r_{12}$ :

$$
d P=n^{2} d V_{1} d V_{2}\left[1+\xi\left(r_{12}\right)\right] .
$$

At small separations, $\xi$ is well approximated as a power law (Groth and Peebles 1986 and references therein),

$$
\begin{aligned}
\xi & =\left(r_{o} / r\right)^{\gamma}, r<10 h^{-1} \mathrm{Mpc}, \\
r_{o} & =5.4 \pm 1 h^{-1} \mathrm{Mpc}, \gamma=1.77 .
\end{aligned}
$$


A useful measure of clustering is the variance of the galaxy count in a randomly placed sphere of radius $r$. The variance divided by the square of the mean value of the count in the sphere is

$$
\left[\frac{\delta N}{N}\right]^{2}=\frac{1}{N}+\int_{V} \frac{d^{2} V}{V^{2}} \xi\left(r_{12}\right) \sim \frac{4 \pi J_{3}(r)}{V},
$$

where

$$
J_{3}(r)=\int_{0}^{r} r^{2} d r \xi(r)
$$

The last equation follows if the mean number $N$ is large and $\xi$ is small or close to flat at $r_{12} \sim r$. The integral $J_{3}$ is estimated to be (Clutton-Brock and Peebles 1981; Davis and Peebles 1983)

$$
J_{3}\left(30 h^{-1} \mathrm{Mpc}\right) \sim 800 e^{ \pm 0.2} h^{-3} \mathrm{Mpc}^{3} \text {. }
$$

If the galaxy two-point correlation function were negligibly small at larger separations, then we see from equations (4) and (6) that the rms fluctuations in galaxy counts averaged over the Hubble length $c / H$ and one tenth the Hubble length are

$$
\begin{aligned}
\frac{\delta N}{N} & =0.0003 \text { at } r=3000 h^{-1} \mathrm{Mpc}, \\
& =0.01 \text { at } r=300 h^{-1} \mathrm{Mpc} .
\end{aligned}
$$

\section{FLUCTUATIONS IN THE OPTICAL BACKGROUND}

The known source of the extragalactic optical background radiation is the population of ordinary galaxies. Because the spectra of galaxies fall off to the blue, the cosmological redshift limits the dominant contribution to the background at wavelength $\lambda \sim 5000 \AA$ to galaxies at redshifts $z \sim 0.5$ or less, so only modest extrapolations are needed to relate the fluctuations in sky brightness due to galaxies to the observed spatial clustering of galaxies at low redshifts. There may be an appreciable contribution to the optical background from young blue galaxies in the phase of heavy element production, if this occurs at redshifts $z<4$. (At $z \sim 4$ the light from massive stars, which peaks at $\lambda \sim 1000 \AA$, would be redshifted to the middle of the optical band. Energy density released at redshift $z$ is reduced by the factor $(1+z)^{4}$; as discussed in section 5 below, if $z \sim 4$, that would make the present background from element production comparable with that produced by normal galaxies.) There may also be a significant contribution from stars between the known galaxies. This is suggested by the fact that, if Newtonian mechanics is adequate for systems of galaxies, most of the mass in the universe is outside the bright central parts. If this dark mass is a population of low mass stars, it could contribute to the optical sky brightness. This and the light from young galaxies could be detected as an excess in the mean sky brightness, as discussed by Mattila (1989, this volume), or as a suppression in the fluctuations in surface brightness across the sky from that expected from the known clustering of sources in ordinary galaxies.

If the optical sky brightness is in fact dominated by starlight from low redshift galaxies, we can compute the autocorrelation function of the sky brightness from the galaxy two-point correlation function. The sky surface brightness $i_{v}$ at frequency $v$ (energy flux per steradian and frequency interval) is a line integral of the luminosity density:

$$
i_{v}=\int_{0}^{t_{o}} c d t \frac{j\left(t, v a_{o} / a\right)}{4 \pi}\left[\frac{a(t)}{a_{o}}\right]^{3} .
$$


The redshift factor is $1+z=a_{o} / a(t)$, where $a(t)$ is the expansion factor at world time $t$, and $a_{o}$ is the present value, at time $t_{o}$. The last factor in equation (8) takes account of the dilution of monochromatic surface brightness by the expansion of the universe. The luminosity density $j_{v}$ (rate of energy release per unit volume and frequency interval) of galaxies can be approximated as a sum of pointlike terms,

$$
j(\mathbf{r})=\sum_{i} L_{i} \delta\left(\mathbf{r}-\mathbf{r}_{\mathrm{i}}\right),
$$

with autocorrelation function

$$
\begin{aligned}
\left\langle\delta j\left(\mathbf{r}_{1}\right) \delta_{j}\left(\mathbf{r}_{2}\right)>\right. & =\left\langle\left(j\left(\mathbf{r}_{1}\right)-<j>\right)\left(j\left(\mathbf{r}_{2}\right)-<j>\right)>\right. \\
& =n<L^{2}>\delta\left(\mathbf{r}_{1}-\mathbf{r}_{2}\right)+n^{2}<L>^{2} \xi\left(\mathbf{r} r_{12}\right)
\end{aligned}
$$

The first term approximates the mean convolution of the luminosity distribution within a galaxy. On using this expression in equation (8), with the power law approximation for $\xi$ in equation (3), one finds that the autocorrelation function of the sky brightness is of the form

$$
<\delta i_{v}(1) \delta i_{v}(2)>=A \delta\left(\vec{\theta}_{1}-\vec{\theta}_{2}\right)+B\left|\vec{\theta}_{1}-\vec{\theta}_{2}\right|^{1-\gamma} \text {. }
$$

The coefficient of the shot noise part arising from the light concentration within individual galaxies is

$$
A=\frac{n_{o} c}{16 \pi^{2} a_{o}^{2}} \int_{0}^{t_{o}} \frac{\left\langle L^{2}>d t\right.}{x(t)^{2}} \frac{a}{a_{o}}
$$

where $x(t)$ is the coordinate distance to a galaxy seen at epoch $t$. This integral diverges at $t \rightarrow t_{o}$, where $x \alpha t_{o}-t$, which means the shot noise is dominated by the nearest galaxies. The coefficient of the extended part of equation (10) is

$$
B=\frac{c n_{o}^{2}}{16 \pi^{2}} \int_{-\infty}^{\infty} \frac{d z}{\left(z^{2}+1\right)^{\gamma / 2}} \int_{0}^{t_{o}} d t<L\left(v a_{o} / a(t), t\right)>^{2}[a(t) x(t)]^{1-\gamma} r_{o}(t)^{\gamma} .
$$

This time integral is well behaved at $t \rightarrow t_{o}$, and because the spectrum $L_{\mathrm{v}}$ decreases with increasing $v$ in typical bright galaxies, the integral is strongly suppressed beyond redshift $z \sim 0.5$. The approximation of the time integral is discussed in Peebles (1980); the order of magnitude result is

$$
\frac{\left(<\delta i_{v}(1) \delta i_{v}(2)>\right.}{\left.<i_{v}\right\rangle^{2}} \sim(0.06)^{2}\left[\frac{\theta_{o}}{\theta}\right]^{\gamma-1},
$$

where the angular coherence length is

$$
\theta_{o}=H r_{o} / c-6^{\prime} .
$$

It appears that the best measurement of the autocorrelation function of the optical background is that of Shectman (1974), who used stacked photographic plates. His results are consistent with the conventional estimates of the mean luminosity per unit volume due to galaxies and of the galaxy clustering length $r_{o}$. That is, there is no evidence either for galaxy clustering on large scales beyond that in equation (7) or for an appreciable contribution to the optical background from sources other than ordinary galaxies. A similar conclusion for the UV $1400-1900 \AA$ background is reported by Martin and Bowyer (1989) and Bowyer $(1989$, this volume). Boughn, Saulson, and Uson (1986) obtained bounds on the fluctuations in the infrared sky brightness at $2.2 \mu \mathrm{m}$ that usefully constrain the light from young galaxies and approach the level needed to detect the fluctuations in light from normal galaxies. 
More detailed measurements of the fluctuations in the optical extragalactic background using the panoramic photon detectors now available would appear to be feasible. It would be of considerable interest to know how the fluctuation spectrum varies with wavelength, as a test for the presence of sources other than ordinary galaxies, and to have improved measures of the fluctuation spectrum as a function of angular scale, as a probe of the character of the galaxy space distribution.

\section{BOUNDS ON LARGE-SCALE MASS FLUCTUATIONS}

Galaxies cluster strongly on small scales, and the observed motions of galaxies in groups and clusters tell us there are roughly comparable small-scale fluctuations in the mass distribution. The relation between this clustering and the primeval seeds out of which it grew is complicated by the extreme nonlinearity. Mass fluctuations on larger scales, where the contrast $\delta M / M$ is small, are more easily interpreted, but the observational measures are considerably poorer. As reviewed here, there are several ways by which measurements of anisotropies in the radiation backgrounds constrain large-scale mass fluctuations and theories of galaxy formation. For other discussions see Rees (1980), Bond (1988a), and references therein.

\subsection{The Aether Drift}

As Strukov describes (1989), the 3K cosmic fireball background radiation (the CBR) has a dipole anisotropy consistent with a motion of the Local Group of galaxies at $600 \mathrm{~km} \mathrm{~s}^{-1}$ relative to the rest frame defined by the CBR. Another possible interpretation is that the dipole is produced by departures from homogeneity and isotropy on scales larger than the Hubble length $c H^{-1}=3000 h^{-1} \mathrm{Mpc}$, but in that case one would expect that dynamical perturbations to the quadrupole terms in the CBR anisotropy are no smaller than the dipole, contrary to what is observed. An isocurvature perturbation, where the primeval mass density is homogeneous but the entropy per baryon, $s$, is a function of position, could produce a pure dipole by suitable choice of the function $s(\mathbf{x})$, but that seems to be a contrived arrangement. Thus, the straightforward and generally accepted interpretation is that the Local Group has a peculiar motion, produced by the gravitational acceleration of mass fluctuations on scales less than the Hubble length.

This velocity interpretation of the CBR dipole might be checked in several ways. The motion would produce a dipole in the $\mathrm{X}$-ray background (called, in this connection, the Compton-Getting effect). Boldt (1989) concludes that the data are not yet adequate for a convincing test for this effect in the X-ray background.

The velocity interpretation also would predict that our motion relative to the mean provided by distant galaxies agrees with that defined by the CBR. The evidence, summarized by Aaronson et al. $(1986,1989)$, is that velocities defined relative to the CBR and galaxy frames of reference agree to better than $\sim 5 \%$ of the cosmological redshift of galaxies at distances greater than $\mathrm{Hr} \sim 4000 \mathrm{~km} \mathrm{~s}^{-1}$. Aaronson et al. (1986) conclude that the rest frame defined by clusters of galaxies in the Arecibo declination range at redshifts 4000 to $10,000 \mathrm{~km} \mathrm{~s}^{-1}$ agrees with the CBR frame to one standard deviation $\sim 200 \mathrm{~km} \mathrm{~s}^{-1}$. This indicates that the motion of the Local Group relative to a universal reference frame has been detected, at $\sim 3$ standard deviations, and that the coherence length of the peculiar velocity field is less than the typical distance to clusters in this sample, that is, below $\sim 40 h^{-1} \mathrm{Mpc}$.

Direct evidence of gravitationally induced motion would be the identification of the mass concentrations responsible for the motion (perhaps to be called the "ultimate attractor," after the "great attractor" of Lynden-Bell et al. 1988; though the gravitational field need not be that of a single mass). If galaxies trace the large-scale mass distribution, and the mean mass density is somewhere between that indicated by local dynamical estimates and the Einstein- 
de Sitter value ( $\Omega$ between -0.1 and 1 ), then the peculiar gravitational acceleration at the Local Group that originates in mass fluctuations within distances $\mathrm{Hr} \sim 4000 \mathrm{~km} \mathrm{~s}^{-1}$ produces a local peculiar motion with at least roughly the right magnitude and direction (Lynden-Bell and Lahav 1988; Yahil 1988, and references therein). This suggests that the bulk of the ultimate attractor lies within a distance $H r \sim 4000 \mathrm{~km} \mathrm{~s}^{-1}$. If this were typical, the coherence length $r_{v}$ of the peculiar velocity field, where the scalar velocity autocorrelation function,

$$
v_{c}(r)^{2}=\left\langle\mathbf{v}\left(\mathbf{r}_{1)} \cdot \mathbf{v}\left(\mathbf{r}_{1}+\mathbf{r}\right)\right\rangle\right. \text {, }
$$

is half its value at zero lag, would be $r_{v} \sim 40 h^{-1} \mathrm{Mpc}$.

To summarize, the evidence is that the CBR defines a universal rest frame relative to which the Local Group is moving at $-600 \mathrm{~km} \mathrm{~s}^{-1}$, and that the coherence length of the peculiar velocity field is $\sim 40 h^{-1} \mathrm{Mpc}$.

\subsection{Mass Fluctuations on the Scale $-300 \mathrm{~h}^{-1} \mathrm{Mpc}$}

We can bound mass fluctuations on scales larger than $r_{v}$ by the limit on their effect on the peculiar velocity field. In linear perturbation approximation, the scalar autocorrelation function of the velocity field produced by the gravitational acceleration of the fluctuating mass distribution is (Peebles 1980)

$$
v_{c}\left(r_{12}\right)^{2}=4 \pi H^{2} \Omega^{1.2} \int_{0}^{\infty} d k P_{k} \sin (k r) /(k r),
$$

where the spectrum $P_{k}$ of mass fluctuations is the transform of the mass autocorrelation function,

$$
\xi_{\rho}(r)=\int d^{3} k P_{k} \mathrm{e}^{i \vec{k} \cdot \vec{r}}
$$

If $\xi_{\rho}$ is negligibly small on large scales, the spectrum $P_{k}$ is nearly flat, and equations (15) and (16) give at large $r$

$$
\begin{gathered}
v_{c}(r)^{2} \sim H^{2} \Omega^{1.2} J_{\rho}(r) / r, \\
J_{\rho}(r)=\int_{0}^{r} r^{2} d r \xi_{\rho}(r) .
\end{gathered}
$$

This relates the large-scale peculiar velocity field and large-scale mass fluctuations as determined by $J_{\rho}$ through equation (4).

If we approximate $J_{\rho}$ by $J_{3}$ in equation (6), we get

$$
v_{c}\left(40 h^{-1} \mathrm{Mpc}\right) \sim 450 \Omega^{0.6} \mathrm{~km} \mathrm{~s}^{-1} \text {. }
$$

This is at least roughly consistent with a velocity clustering length [at which $v_{c}^{2}$ is half its central value of perhaps $\sim\left(600 \mathrm{~km} \mathrm{~s}^{-1}\right)^{2}$ ] of $r_{v} \sim 40 h^{-1} \mathrm{Mpc}$.

Now let us use equation (17) to bound mass fluctuations on larger scales. Taking $r \sim 300 h^{-1} \mathrm{Mpc}$, and using $v_{c}<600 \mathrm{~km} \mathrm{~s}^{-1}$, we get a bound on $J_{\mathrm{p}}$ integrated to this larger radius. This in equation (4) gives

$$
\frac{\delta M}{M} \leq 0.03 \Omega^{-0.6} \quad \text { at } \quad r=300 h^{-1} \mathrm{Mpc} .
$$

Another limit comes from the isotropy of the X-ray background. As discussed by Setti (1989, this volume), an appreciable fraction of the X-ray background comes from clusters and active galaxies at redshifts less than unity. If the space density of these sources fluctuates by the fractional amount $\delta N / N$ on the scale $r$, it produces surface brightness fluctuations in the $\mathrm{X}$-ray background, 


$$
\frac{\delta i}{i} \sim f\left(\frac{H r}{c}\right]^{1 / 2} \frac{\delta N}{N}
$$

on the angular scale

$$
\theta \sim \mathrm{Hr} / \mathrm{c},
$$

where $f$ is the fraction of the background originating at $z \sim 1$ or less. At $r \sim 300 h^{-1} \mathrm{Mpc}$, the angular scale is $\theta \sim 6^{\circ}$, the HEAO-1 A2 bound is $\delta i / i \leq 0.03$ (Boldt 1989), and the resulting bound on the rms density fluctuation is

$$
\frac{\delta N}{N} \leq 0.1 f^{-1} \quad \text { at } \quad r=300 h^{-1} \mathrm{Mpc} .
$$

For $\Omega \sim 0.1$, equations (19) and (22) allow large-scale mass fluctuations considerably larger than equation (7), the value expected if galaxy positions were uncorrelated at separations greater than $-30 h^{-1} \mathrm{Mpc}$. That is, we cannot exclude the possibility that the mass fluctuation power spectrum on the scale of a tenth the Hubble length is considerably larger than a flat extrapolation from equation (6). In the baryon isocurvature theory, the mass fluctuation spectrum has a considerable bulge at $r \sim 300 h^{-1} \mathrm{Mpc}$ (Peebles 1987). Tighter constraints on such large-scale fluctuations would be of considerable interest. Perhaps they will be obtained from measurements of fluctuations in the optical background, by integrated surface brightness measurements or galaxy counts.

\subsection{Mass Fluctuations on the Scale of the Hubble Length}

Sachs and Wolfe (1967) pointed out that inhomogeneities in the mass distribution gravitationally perturb the CBR: the radiation moves through an inhomogeneous gravitational potential field that shifts the frequency and hence the surface brightness of the radiation. The effect is most sensitive to mass fluctuations on the scale of the Hubble length, because for given relative mass fluctuation $\delta M / M$, the perturbation to the potential is larger the larger the scale of the fluctuation. The following review of bounds on mass fluctuations from the Sachs-Wolfe effect assumes an Einstein-de Sitter model universe, because that greatly simplifies the analysis.

It is convenient to express the CBR temperature as a function of position across the sky in the spherical harmonic expansion

$$
\frac{\delta T}{T}=\sum a_{l}^{m} Y_{l}^{m}(\theta, \phi) .
$$

If the temperature fluctuations approximate an isotropic random process, the expected values of the expansion coefficients $a_{l}^{m}$ are independent of $m$ :

$$
\left.a_{l}=\left(<\left|a_{l}^{m}\right|^{2}\right\rangle\right)^{1 / 2} \text {. }
$$

In this notation, the bound on the quadrupole moment of the CBR from the RELIKT experiment discussed by Strukov (1989) is

$$
a_{2} \leq 5 \times 10^{-5}
$$

In an Einstein-de Sitter universe, the expected values of the $a_{l}$ are given by the equation (Peebles 1981, 1982)

$$
\left(a_{l}\right)^{2}=4 \pi^{2} H^{4} \int_{0}^{\infty} k^{-2} d k P_{k}\left[j_{l}\left(2 k c / H a_{o}\right)\right]^{2},
$$

where $j_{l}$ is the spherical Bessel function, and $P_{k}$ is the power spectrum of the mass fluctuations, related to the mass autocorrelation function $\xi_{\rho}$ by equation (16). (To be more precise, $\xi_{\rho}$ is the autocorrelation function of the growing mode of the density perturbation in synchronous 
time-orthogonal gauge; equation (25) is derived in linear perturbation theory following $\$ 93$ of Peebles 1980.) If the mass autocorrelation function vanishes at large separations, then the integral $J_{\rho}$ (equation [17]) converges to a fixed value, and equation (26) gives the expected quadrupole moment,

$$
a_{2}=\left(\frac{4 \pi J_{\rho} H^{3}}{105 c^{3}}\right)^{1 / 2} .
$$

If galaxies trace the large-scale mass distribution, then $J_{\mathrm{p}} \sim J_{3} \sim 800 h^{-3} \mathrm{Mpc}^{3}$ from equation (6), and we get

$$
a_{2} \sim 6 \times 10^{-5} \text {. }
$$

Given the uncertainties in the theoretical estimate, this white noise case is just possible, but we certainly cannot imagine that the mass fluctuation spectrum on the scale of the Hubble length is significantly larger than a flat extrapolation from $\sim 30 h^{-1} \mathrm{Mpc}$. For example, if the mass autocorrelation function at large separations varied as $\xi_{\rho} \propto r^{-2}$, which corresponds to $P_{k} \propto k^{-1}$, then the predicted quadrupole moment would be

$$
\left(a_{2}\right)^{2}=\pi H^{2} r^{2} \xi_{\rho}(r) / 18 c^{2}
$$

In the biasing picture (discussed by Kaiser and Lahav 1988; Davis and Efstathiou 1988, and references therein), the mass autocorrelation function at $h r \sim 10 h^{-1} \mathrm{Mpc}$ is smaller than the galaxy two-point function $\xi(r)$ (equation [3]) by a factor $\sim 4$. With this factor, and matching correlation functions at $h r=10 \mathrm{Mpc}$, we get $a_{2} \sim 2 \times 10^{-4}$, which is ruled out. Consistent with this, the direct estimates of the galaxy two-point correlation function $\xi(r)$ indicate that the function falls below the small-scale power law at $h r \sim 10 \mathrm{Mpc}$ (Groth and Peebles 1986).

To summarize, the evidence from the observed relative velocities of galaxies and the velocity interpretation of the CBR dipole is that the mass fluctuation spectrum is roughly comparable with that of the observed galaxy clustering on scales less than about $30 \mathrm{~h}^{-1} \mathrm{Mpc}$. The spectrum extrapolated from this scale to the Hubble length must be flat or decreasing toward larger scales, to avoid overproducing the low-order multipole moments of the CBR. This is consistent with the scale-invariant Zel'dovich spectrum $P_{k} \propto k$ produced in the simplest inflation models (Bardeen, Steinhardt, and Turner 1983). The same is true of the explosion picture (Ostriker 1986), and some versions of the baryon isocurvature picture, depending on how the spectrum of fluctuations in the primeval entropy per baryon is adjusted (Peebles 1987). On the other hand, it is still possible that the quadrupole moment $a_{2}$ will be found to be consistent with equation (27). In this case the simplest interpretation would be that the large-scale structure of the universe developed out of uncorrelated adiabatic mass density fluctuations.

\section{SCATTERING OF THE CBR IN YOUNG GALAXIES}

Measurements of small angular scale fluctuations in the CBR constrain theories for the origin of galaxies and groups and clusters of galaxies. The case where the CBR has not been appreciably scattered since decoupling of matter and radiation at redshift $z-1000$ has been analyzed in detail (Bond $1988 b$ and references therein). Another possibility is that stars form and ionize matter at high redshifts, in which case the CBR is scattered by free electrons and the fluctuations in the CBR left over from decoupling are replaced by perturbations caused by the scattering. The effect of scattering by moving plasma is studied in second order perturbation theory by Vishniac (1987) and Efstathiou and Bond (1987). In the other limiting case to consider, the CBR is scattered by plasma already concentrated in discrete compact clouds: young galaxies or pre-galaxy star clusters. This is an interesting case because the clouds could 
provide the stars to ionize the matter. The order-of-magnitude estimates presented here indicate that the predicted fluctuations in this case are below the experimental bounds.

Since the CBR is last scattered at an epoch when the value of the density parameter is close to unity, we can approximate the relation between cosmic time and redshift as

$$
t=\frac{2}{3 \Omega^{1 / 2} H}(1+z)^{-3 / 2},
$$

where $\Omega$ is the present value of the density parameter. The mean number of times a photon is scattered at redshift $<z$ is the time integral of the scattering probability per unit time, $\sigma_{T} n(t) c$, which with equation (29) is

$$
\tau_{b}(x)=\frac{2 \sigma_{T} n_{o} c}{3 H \Omega^{1 / 2}}\left[(1+z)^{-3 / 2}-1\right],
$$

with $\sigma_{T}$ the Thomson scattering cross section. This amounts to $\tau_{b}=0.5$ at redshift

$$
1+z_{1 / 2}=\left[1+\frac{10.8 \Omega^{1 / 2}}{x \Omega_{B} h}\right]^{2 / 3},
$$

where $\Omega_{B}$ is the density parameter in baryons and $x$ is the fraction of the baryons in optically thin ionized gas. In the biased cold dark matter picture (Davis and Efstathiou 1988, and references therein), where standard choices of the cosmological parameters are $h=0.5, \Omega=1$, and $\Omega_{b} \sim 0.1$, the characteristic epoch of last scattering is $z_{1 / 2} \sim 35$. In this theory, density fluctuations would be small at $z_{1 / 2}$, so it is reasonable to assume the CBR has not been scattered since decoupling. On the other hand, with the current estimate $h=0.67$ of van den Bergh (1989), and the density parameter indicated by dynamical estimates, $\Omega=\Omega_{B} \sim 0.1$, one finds $z_{1 / 2} \sim 13$. It is quite conceivable that plasma-rich young galaxies or pre-galaxy star clusters existed at this epoch and that they scattered the CBR.

Assuming such objects existed, with scattering probability $\tau$ per cloud, their perturbation to the CBR temperature along a line of sight would be

$$
\frac{\delta T}{T} \sim \tau \frac{v_{1}}{c}+(1-\tau) \tau \frac{v_{2}}{c}+(1-\tau)^{2} \tau \frac{\nu_{3}}{c}+\ldots
$$

The velocity in the $n^{\text {th }}$ cloud along the line of sight is $v_{n}$, and the prefactor is the probability that the last scattering is in the $n^{\text {th }}$ cloud counting back from the present. Squaring this expression, averaging, and ignoring the correlation of velocities of clouds, we get

$$
\left(\frac{\delta T}{T}\right)^{2}=\Sigma \tau^{2}(1-\tau)^{2 n}(v / c)^{2},
$$

which gives

$$
\frac{\delta T}{T}=\left[\frac{\tau}{2-\tau}\right]^{1 / 2} \frac{v}{c} .
$$

For an order-of-magnitude estimate, let us suppose a typical plasma cloud has the mass and density characteristic of the bright part of a large galaxy, radius $r \sim 5 h^{-1} \mathrm{kpc}$ and circular velocity $v_{c}-250 \mathrm{~km} \mathrm{~s}^{-1}$. This works out to surface density $\tau \sim 0.08$ if the bulk of the matter is dilute ionized gas. The rms line-of-sight velocity due to internal motion is $\sim v_{c} / 3^{1 / 2}$. These numbers in equation (34) give

$$
\delta T / T \sim 1 \times 10^{-4} .
$$


The temperature fluctuation would be coherent over the angular size of one of the plasma clouds,

$$
\theta \sim\left(1+z_{1 / 2}\right) \Omega H r /(2 c) \sim 3 \Omega \operatorname{arcsec},
$$

for $z_{1 / 2} \sim 20$. This effect is well below the experimental bounds summarized by De Zotti (1989, this volume).

The effect of correlated motions of the gas clouds can be roughly estimated by scaling the velocity field discussed in section 3.1 back in time to the epoch $z_{1 / 2}$ of last scattering. Let us model the present large-scale velocity field as adjacent patches of width $w \sim 40 h^{-1} \mathrm{Mpc}$ within each of which the velocity is roughly uniform at rms value $\sim 600 \mathrm{~km} \mathrm{~s}^{-1}$. In linear perturbation theory, the velocity scales with time as $\sim(1+z)^{-1 / 2}$, which brings the onedimensional $\mathrm{rms}$ value of the velocity at $z_{1 / 2} \sim 20$ to $\sim 100 \mathrm{~km} \mathrm{~s}^{-1}$. The net perturbation to the CBR depends on the correlation of peculiar motions of neighboring patches, as discussed by Efstathiou (1988). Here, because the probability that a line of sight intersects a galaxy in a given patch is fairly small, it is a reasonable approximation to ignore this correlation.

The scattering probability through the width $w$ of a coherent patch is

$$
\tau \sim 9 \times 10^{-4} \Omega_{B} h\left(1+z_{1 / 2}\right)^{2} \sim 0.02
$$

for $\Omega_{B} \sim 0.1, h=0.67$, and $z_{1 / 2} \sim 20$. These numbers give

$$
\frac{\delta T}{T} \sim 3 \times 10^{-5} \text { at } \theta \sim \frac{H w \Omega}{2 c} \sim 2 \operatorname{arc} \min ,
$$

for $\Omega \sim 0.1$. This is just at the limit from the Owens Valley Radio Observatory (Readhead et al. 1989).

The conclusion is that one can find gravitational instability scenarios in which fluctuations in the CBR associated with the formation of galaxies or pre-galaxy star clusters at high redshift are consistent with present experiments. This early galaxy formation picture is constrained by the CBR anisotropy on scales of arcminutes, which would be produced by the large-scale velocity field. More detailed calculations of this effect will be of interest.

\section{BACKGROUND RADIATION FROM ELEMENT PRODUCTION}

A signature of the electromagnetic radiation released in the production of the heavy elements in galaxies may be the fluctuations in the background due to the clustered distribution of the sources in galaxies. This heavy element radiation background (HERB) could reach us directly, as redshifted starlight. The other commonly discussed possibility is that the HERB is dominated by thermal radiation from dust that has absorbed the starlight, a case that has attracted considerable attention with the possible detection of a submillimeter radiation background significantly in excess of a $2.7 \mathrm{~K}$ Planck spectrum (Matsumoto et al. 1988; Draine and Shapiro 1989; Bond, Carr, and Hogan 1989, and references therein). Starlight could be absorbed within the galaxy that produced it (as would be the case in our galaxy if the amount of dust were $\sim 3$ times higher in the past) or, in the other limit, starlight may have traveled many galaxy clustering lengths before being absorbed by dust in galaxies. In all the above cases, the autocorrelation function of the HERB source luminosity density is the same as the galaxy correlation function. As discussed by Bond, Carr, and Hogan (1989), this would imply a significant HERB anisotropy. I present here a review of the effects that yield the order of magnitude of the anisotropy.

A simple and reasonable model for the source density of this background is based on the following assumptions. Suppose a fraction $f$ of the baryon mass has been converted to radiation at a uniform rate ending at the epoch $t_{e}$. Then the mean luminosity density is 


$$
j=\frac{\rho_{o} c^{2} f}{t_{e}}\left[\frac{a_{o}}{a}\right]^{3}, \quad z>z_{e},
$$

where $\rho_{o}$ is the present mean baryon mass density and $z_{e}$ is the redshift at the end of the epoch of build-up of the heavy elements. As we have seen, it is reasonable to assume that the HERB source density autocorrelation function is

$$
\left\langle j_{1} j_{2}\right\rangle=\langle j\rangle^{2}\left[1+\xi\left(r_{12}\right)\right] .
$$

The shot noise term (analogous to the first term on the right-hand side of equation [10]) is subdominant if, as will be assumed, $t_{e}$ is much less than the present epoch. To simplify the discussion, I will suppose that the galaxy two-point correlation function $\xi$ is negligibly small at $r>30 h^{-1} \mathrm{Mpc}$, and that the integral of $\xi$ varies with time as

$$
a_{o}^{3} \int_{0}^{\infty} x^{2} d x \xi=J_{3}\left(a / a_{o}\right)^{2},
$$

where $x$ is a comoving coordinate, $J_{3}$ is given by equation (6), and last factor assumes an Einstein-de Sitter universe with galaxies clustered with mass. If the density were lower than Einstein-de Sitter, or galaxies more strongly clustered than mass, the clustering at high redshift would be higher, increasing the expected HERB anisotropy. Generalization of the calculation to these cases and to the case of large-scale correlations of galaxy positions is left as exercise.

The mean HERB surface brightness integrated over all frequencies is

$$
i_{h}=\int_{0}^{t_{o}} c d t(j / 4 \pi)\left[a(t) / a_{o}\right]^{4},
$$

the last factor in the integral accounting for the dimunition in bolometric surface brightness by the expansion of the universe. With equation (37) this is

$$
i_{h}=\frac{3}{20 \pi} \frac{\rho_{o} c^{3} f}{1+z_{e}} .
$$

The HERB autocorrelation function is

$$
\begin{aligned}
c(\theta) & =\left\langle\delta i_{1} \delta i_{2}>\right. \\
& =\int_{0}^{t_{0}} \frac{c d t}{16 \pi^{2}} \int_{-\infty}^{\infty} d u j(t)^{2} \xi\left(t, r_{12}\right)\left[a(t) / a_{o}\right]^{8} .
\end{aligned}
$$

The physical separation of the points in $\xi$ is

$$
r_{12}=\left(a^{2} x^{2} \theta^{2}+u^{2}\right)^{1 / 2}
$$

where the coordinate distance can be approximated as $x \sim 2 c /\left(H a_{o} \Omega\right)$. The mean square fluctuation in the background smoothed through a window of radius $\theta$ is

$$
\begin{aligned}
(\Delta i)^{2} & =\int d \Omega_{1} d \Omega_{2} c\left(\theta_{12}\right) /\left(\pi \theta^{2}\right)^{2} \\
& =\frac{1}{\pi \theta^{2}} \int d \Omega_{1} c\left(\theta_{1}\right),
\end{aligned}
$$

where the second line follows if the window is large enough to include the significant correlation of the HERB sources. On writing the proper spatial volume element as

$$
a^{3} d^{3} x=(a x)^{2} d \Omega d u,
$$

and using equation (39) for the integral of $\xi$ and equation (37) for the mean luminosity density 
as a function of time, we get the mean square fluctuation in the background due to the energy release in heavy element production,

$$
(\Delta i)^{2}=\frac{9 \rho_{o}^{2} c^{6} f^{2}}{416 \pi^{2} \theta^{2}} \frac{J_{3} H^{3}}{c^{3}}\left[\frac{a_{e}}{a_{o}}\right]^{7 / 2} \Omega^{5 / 2} .
$$

With equation (41), the fractional fluctuation in the HERB is

$$
\frac{\Delta i}{i}=\left[\frac{25}{26}\right]^{1 / 2} \frac{\Omega^{5 / 4}}{\theta}\left[\frac{J_{3} H^{3}}{c^{3}}\right]^{1 / 2}\left[\frac{a_{e}}{a_{o}}\right]^{3 / 4} \sim \frac{0.6 \Omega^{5 / 4}}{\left(1+z_{e}\right)^{3 / 4} \theta_{\operatorname{arcmin}}} .
$$

This assumes the smoothing window radius $\theta$ subtends a length large compared with the source clustering length, which translates to

$$
\theta>>\frac{H r_{o} \Omega}{2 c\left(1+z_{e}\right)^{2 / 3}} \geq 3 \Omega \operatorname{arcmin} .
$$

Detectability of the HERB fluctuations depends on the wavelength. If the HERB were starlight from galaxies at modest redshifts, the fluctuations would be subdominant to the contribution from ordinary low redshift galaxies, as discussed in section 2 . If the HERB were thermal radiation from dust, it would be concentrated to the Wein part of the CBR spectrum (Wright and Peterson 1989; Bond Carr and Hogan 1989). Orders of magnitude in this case are as follows.

If $\sim 3 \%$ of baryon density $\Omega_{B} \sim 0.03$ is converted to heavy elements at $z_{e} \sim 10$, the mean background (equation [41]) is

$$
i \text { (HERB) } \sim 7 \times 10^{-6} \mathrm{erg} \mathrm{cm}^{-2} \mathrm{~s}^{-1} \mathrm{sr}^{-1} .
$$

If the HERB is reradiated by dust, it would appear at wavelength $\lambda \leq 600 \mu$, where the CBR flux density is

$$
\begin{aligned}
i(C B R<\lambda) & -2 k T c \lambda^{-3} \mathrm{e}^{-h c / k T \lambda} \\
& \sim 2 \times 10^{-5} \mathrm{erg} \mathrm{cm}^{-2} \mathrm{~s}^{-1} \mathrm{sr}^{-1},
\end{aligned}
$$

which is comparable with the HERB. That is, if the starlight from heavy element production is reradiated by dust in galaxies at modest redshifts, it could produce a substantial signal, comparable with equation (47). The search for fluctuations in the short wavelength part of the CBR spectrum thus will be followed with particular interest.

Acknowledgment-I am grateful to Roman Juszkiewicz for stimulating discussions. This research was supported in part by the U.S. National Science Foundation.

\section{REFERENCES}

Aaronson, M. et al. 1986, Ap. J., 302, 536.

Aaronson, M. et al. 1989, Ap. J., 338, 654.

Bardeen, J., Steinhardt, P. J., and Turner, M. S. 1983, Phys. Rev., D28, 679.

Boldt, E. 1989, in Berkeley Workshop on Particle Astrophysics, Berkeley, December 8, 1989.

Bond, J. R. 1988a, in Large-Scale Motions in the Universe, eds. V. C. Rubin and G. V. Coyne (Princeton: Princeton University Press), p. 419.

Bond, J. R. 1988b, in The Early Universe, eds. W. G. Unruh and G. W. Semenoff (Dordrecht: Reidel), p. 283.

Bond, J. R., Carr, B. J., and Hogan, C. J. 1989, preprint.

Boughn, S. P., Saulson, P. R., and Uson, J. M. 1986, Ap. J., 301, 17.

Bowyer, S. 1989, in Proc. IAU 139, Galactic and Extragalactic Background Radiation, ed. S. Bowyer and Ch. Leinert (Dordrecht: Kluwer Academic Publisher).

Clutton-Brock, M. and Peebles, P. J. E. 1981, Ap. J., 86, 1115. 
Davis, M. and Efstathiou, G. 1988, in Large-Scale Motions in the Universe, eds. V. C. Rubin and G. V. Coyne (Princeton: Princeton University Press), p. 439.

Davis, M. and Peebles, P. J. E. 1983, Ap. J, 267, 465.

Draine, B. T. and Shapiro, P. R. 1989, Princeton Observatory Preprint 310.

Efstathiou, G. 1988, in Large-Scale Motions in the Universe, eds. V. C. Rubin and G. V. Coyne (Princeton: Princeton University Press), p. 299.

Efstathiou, G. and Bond, J. R. 1987, MNRAS, 227, 33P.

Groth, E. J. and Peebles, P. J. E. 1986, Ap. J., 310, 507.

Kaiser, N. and Lahav, O. 1988, in Large-Scale Motions in the Universe, eds. V. C. Rubin and G. V. Coyne (Princeton: Princeton University Press), p. 339.

Lynden-Bell, D. et al. 1988, Ap. J., 326, 19.

Lynden-Bell, D. and Lahav, O. 1988, in Large-Scale Motions in the Universe, eds. V. C. Rubin and G. V. Coyne (Princeton: Princeton University Press), p. 199.

Martin, C. and Bowyer, S. 1989, Ap. J., 338, 677.

Matsumoto, T. et al. 1988, Ap. J., 329, 567.

Mattila, K. 1989, in Proc. IAU, 139, Galactic and Extragalactic Background Radiation, ed. S. Bowyer and Ch. Leinert (Dordrecht: Kluwer Academic Publisher).

Ostriker, J. P. 1986, in Galaxy Distances and Deviations from Universal Expansion, eds. B. F. Madore and R. B. Tully (Dordrecht: Reidel), 273.

Peebles, P. J. E. 1980, Large-Scale Structure of the Universe eds. V. C. Rubin and G. V. Coyne (Princeton: Princeton University Press).

Peebles, P. J. E. 1981, Ap. J., 243, L119.

Peebles, P. J. E. 1982, Ap. J., 263, L1.

Peebles, P. J. E. 1987, Nature, 327, 210.

Readhead, A. et al. 1989, Ap. J, in press.

Rees, M. J. 1980, in Objects of High Redshifts, eds. G. O. Abell and P. J. E. Peebles (Dordrecht: Reidel), 207.

Sachs, R. K. and Wolfe, A. M. 1967, Ap. J, 147, 73.

Setti, G. 1989, in Proc. IAU 139, Galactic and Extragalactic Background Radiaton, ed. S. Bowyer and Ch. Leinert (Dordrecht: Kluwer Academic Publisher).

Shectman, S. A. 1974, Ap. J., 188, 233.

Strukov, I. A. 1989, paper presented at IAU Symposium 139, "Galactic and Extragalactic Background Radiation," June, 12-16, 1989, Heidelberg, Federal Republic of Germany.

van den Bergh, S. 1989, in Astronomy and Astrophysics Review, ed. L. Woltjer.

Vishniac, E. T. 1987, Ap. J., 322, 597.

Wright, G. and Peterson, J. 1989, preprint.

Yahil, A. 1988, in Large-Scale Motions in the Universe, eds. V. C. Rubin and G. V. Coyne (Princeton: Princeton University Press), 219. 